\title{
An Iterative Method for Solving Two Special Cases of Lane-Emden Type Equation
}

\author{
Pedro Pablo Cárdenas Alzate \\ Department of Mathematics, Universidad Tecnológica de Pereira, Pereira R, Colombia \\ Email: ppablo@utp.edu.co
}

Received 18 March 2014; revised 22 April 2014; accepted 8 May 2014

Copyright (C) 2014 by author and Scientific Research Publishing Inc.

This work is licensed under the Creative Commons Attribution International License (CC BY).

http://creativecommons.org/licenses/by/4.0/

(c) (i) Open Access

\section{Abstract}

In this work we apply the differential transformation method or DTM for solving some classes of Lane-Emden type equations as a model for the dimensionless density distribution in an isothermal gas sphere $\left(y^{\prime \prime}+\frac{2}{x} y^{\prime}+\mathrm{e}^{ \pm y}=0\right)$ and as a study of the gravitational potential of (white-dwarf) stars $\left(y^{\prime \prime}+\frac{2}{x} y^{\prime}+\left(y^{2}-C\right)^{3 / 2}=0\right)$, which are nonlinear ordinary differential equations on the semi-infinite domain [1] [2]. The efficiency of the DTM is illustrated by investigating the convergence results for this type of the Lane-Emden equations. The numerical results show the reliability and accuracy of this method.

\section{Keywords}

Differential Transformation, Lane-Emden, Isothermal Gas Sphere, White-Dwarfs, Iterative Method

\section{Introduction}

Other classical nonlinear equation, which has been the object of much study, is Lane-Emden's equation. This equation has the form

$$
y^{\prime \prime}+\frac{2}{x} y^{\prime}+g(y)=0
$$

with $0<x \leq 1$ and the subject to initial conditions

$$
y(0)=\alpha, y^{\prime}(0)=\beta
$$


where $\alpha$ and $\beta$ are constants and $g(y)$ is a real-valued continuous function where $\alpha$ and $\beta$ are constants and $g(y)$ is a real-valued continuous function. The Equation (1) was used to model various problems, including the isothermal gas spheres, theory of thermionic currents and the gravitational potential of stars [1] among others.

Let us consider a spherical cloud of gas (see Figure 1) and denote its hydrostatic pressure at a distance $r_{1}$ from the centre by $P$. Let $M\left(r_{1}\right)$ be the mass of the spheres of radius $r_{1}, \phi$ the gravitational potential of the gas and $g$ the acceleration of gravity.

Then, we have the following equation

$$
g=\frac{G M\left(r_{1}\right)}{r_{1}^{2}}=-\phi^{\prime}\left(r_{1}\right)
$$

where $G$ is the gravitational constant.

Thus, three conditions are assumed for the determination of $\phi$ and $P$

$$
\mathrm{d} P=-g \rho \mathrm{d} r_{1}=\rho \mathrm{d} \phi
$$

where $\rho$ is the density of the gas.

$$
\nabla^{2} \phi=\phi^{\prime \prime}\left(r_{1}\right)+\frac{2}{r_{1}} \phi^{\prime}\left(r_{1}\right)=-4 \pi G_{\rho}
$$

and

$$
P=K \rho^{\gamma}
$$

where $\gamma$ and $K$ are arbitrary constants.

Now, solving (4) and (6) with $\phi=0$ when $\rho=0$ we have

$$
\rho=\phi^{\frac{1}{\gamma-1}} K^{\frac{1}{1-\gamma}}
$$

or

$$
\rho=L \phi^{n}
$$

where $n=\frac{1}{\gamma-1}$ and $L=K^{-n}$. If this value of $\rho$ is replaced into Equation (5), we obtain

$$
\nabla^{2} \phi=-\delta^{2} \phi^{n}
$$

where $\delta^{2}=4 \pi L G$.

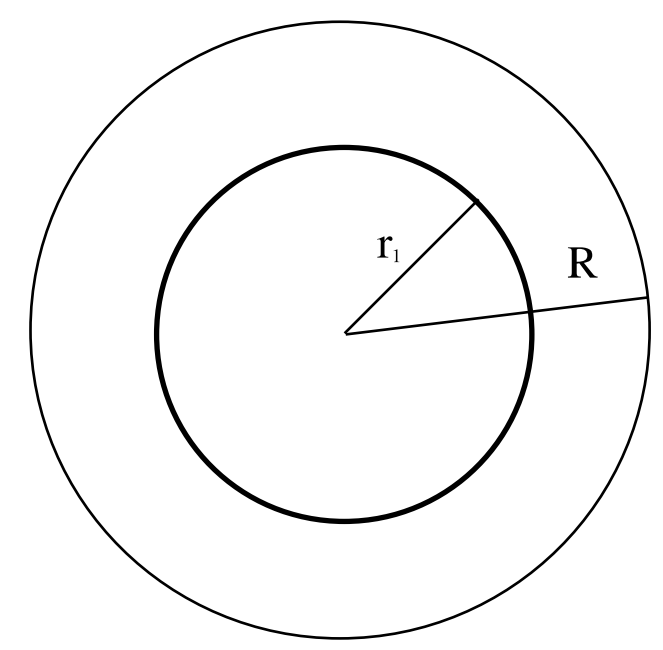

Figure 1. Spherical cloud of gas. 
Now, since $\frac{1}{\rho} \mathrm{d} P=\mathrm{d} \phi$, by integration $\phi=K \log \left(\frac{\rho}{\rho_{0}}\right)$, that is, $\rho=\rho_{0} \mathrm{e}^{\frac{\phi}{K}}$. If $\rho_{0}$ is the central density, then $\phi_{0}$ must be zero, a change from the condition in the previous case where $\phi$ was zero only at the boundary of the sphere.

Poisson's equation is now replaced by

$$
\nabla^{2} \phi=-\delta^{2} \mathrm{e}^{\frac{\phi}{K}}
$$

where $\delta^{2}=4 \pi \rho_{0} G$, equation which is known as Liouville's equation. If we assume symmetry as before, Equation (1) in polar coordinates reduces to the following

$$
\phi^{\prime \prime}\left(r_{1}\right)+\frac{2}{r_{1}} \phi^{\prime}\left(r_{1}\right)+\delta^{2} \mathrm{e}^{\frac{\phi}{K}}=0
$$

which replaces Equation (9).

$$
\begin{aligned}
& \text { If we let } \phi=K y \text { and } r_{1}=\frac{\sqrt{K}}{\delta} x \text {, then (11) becomes } \\
& \qquad y^{\prime \prime}+\frac{2}{x} y^{\prime}+\mathrm{e}^{y}=0
\end{aligned}
$$

which is to solved subject to the boundary conditions $y(0)=0$ and $y^{\prime}(0)=0$. The counterpart [2] of the Equation (12) in which $\mathrm{e}^{y}$ is replaced by $\mathrm{e}^{-y}$ appears in Richardson's theory of thermionic currents when one seeks to determine the density and electric force of an electron gas in the neighborhood of a hot body in thermal equilibrium.

Finally, now consider $g(y)=\left(y^{2}-C\right)^{3 / 2}$, then Equation (1) is turned to the white-dwarf equation, which introduced by [2] in his study of gravitational potential of the degenerate stars. This Equation is defined in the form

$$
y^{\prime \prime}+\frac{2}{x} y^{\prime}+\left(y^{2}-C\right)^{3 / 2}=0
$$

With $x \in[0, \infty]$ and subject to initial conditions $y(0)=1$ and $y^{\prime}(0)=0$. For instance if $C=0$, we have Lane-Emden equation of index $m=3$ [3].

The Differential Transformation Method is a semi-numerical-analytic method for solving ordinary and partial differential equations. Zhou first introduced the concept of DTM in 1986 [4]. This technique constructs an analytical solution in the form of a polynomial. DTM is an alternative procedure for obtaining analytical Taylor series solution of the differential equations. The series often coincides with the Taylor expansion of the true solution at point $x_{0}=0$, in the value case, although the series can be rapidly convergent in a very small region.

Many numerical methods were developed for this type of nonlinear ordinary differential equations, specifically on Lane-Emden type equations such as the Adomian Decomposition Method (ADM) [5], the Homotopy Perturbation Method (HPM) [6] [7], the Homotopy Analysis Method (HAM) [8] and Berstein Operational Matrix of Integration [9]. In this paper, we show superiority of DTM by applying them on the some type LaneEmden type equations. The power series solution of the reduced equation transforms into an approximate implicit solution of the original equation. A spectral method (Legendre-Spectral method) was proposed to solve white-dwarf equation; this spectral method provides the most convenient computer implementation [10].

\section{Description of DTM}

Differential transformation method of the function $y(x)$ is defined as follows

$$
Y(k)=\frac{1}{k !}\left[\frac{\mathrm{d}^{k} y(x)}{\mathrm{d} x^{k}}\right]_{x=x_{0}}
$$

In (13), $y(x)$ is the original function and $Y(k)$ is the transformed function and the inverse differential 
transformation is defined by

$$
y(x)=\sum_{k=0}^{\infty} Y(k) x^{k}
$$

In real applications, function $y(x)$ is expressed by a finite series and Equation (14) can be written as

$$
\sum_{k=0}^{n} Y(k) x^{k}
$$

Equation (15) implies that

$$
\sum_{k=n+1}^{\infty} Y(k) x^{k}
$$

The following theorems can be deduced from Equations (13) and (15).

Theorem 1 If $y(x)=f(x) \pm g(x)$, then $Y(k)=F(k) \pm G(k)$.

Theorem 2 If $y(x)=a f(x)$, then $Y(k)=a F(k), a \in \mathbb{R}$.

Theorem 3 If $y(x)=\frac{\mathrm{d}^{n} g(x)}{\mathrm{d} x^{n}}$, then $Y(k)=\frac{(k+n) !}{k !} G(k+n)$.

Theorem 4 If $y(x)=g(x) h(x)$, then $Y(k)=\sum_{k_{1}=0}^{k} G\left(k_{1}\right) H\left(k-k_{1}\right)$.

Theorem 5 If $y(x)=x^{n}$, then $Y(k)=\delta(k-n)$, where $\delta(k-n)= \begin{cases}1, & k=n \\ 0, & k \neq n\end{cases}$

Theorem 6 (Cárdenas) If $y(x)=x^{n} f(x)$, with $m \in N$, then $Y(k)= \begin{cases}0, & k<m \\ F(k-m), & k \geq n\end{cases}$

The proofs of Theorems are available in [11].

\section{Test Problems}

To illustrate the ability of DTM for the Lane-Emden type equation, three examples are provided. The results reveal that this method is very effective.

Example 1 Consider the nonlinear initial-value problem $y^{\prime \prime}+\frac{2}{x} y^{\prime}+\mathrm{e}^{y}=0$ subject to $y(0)=y^{\prime}(0)=0$. Multiplying both sides by $x$ we obtain

$$
x y^{\prime \prime}+2 y^{\prime}+x \mathrm{e}^{y}=0
$$

Applying theorems 1-6 to Equation (16)

$$
Y(k+1)=\frac{-1}{(k+2)(k+1)}\left[\delta(k-1)+Y(k-1)+\frac{1}{2 !} S_{1}+\frac{1}{3 !} S_{2}+\frac{1}{4 !} S_{3}+\cdots\right]
$$

where

$$
\begin{gathered}
S_{1}=\sum_{k_{1}=0}^{k-1} Y\left(k_{1}\right) Y\left(k-1-k_{1}\right) \\
S_{2}=\sum_{k_{2}=0}^{k-1} \sum_{k_{1}=0}^{k_{2}} Y\left(k_{1}\right) Y\left(k_{2}-k_{1}\right) Y\left(k-1-k_{2}\right) \\
S_{3}=\sum_{k_{3}=0}^{k-1} \sum_{k_{2}=0}^{k_{3}} \sum_{k_{1}=0}^{k_{2}} Y\left(k_{1}\right) Y\left(k_{2}-k_{1}\right) Y\left(k_{3}-k_{2}\right) Y\left(k-1-k_{3}\right)
\end{gathered}
$$

for all $k \geq 1$.

Now, from the initial conditions $y(0)=y^{\prime}(0)=0$ we can obtain

$$
Y(0)=0 \text { and } Y(1)=0
$$

Substituting Equation (21) into Equation (17) and by recursive method, the results are listed as follows. 


$$
\begin{gathered}
\text { For } k=1, Y(2)=\frac{-1}{6}[\delta(1-1)+Y(0)+0+0]=\frac{-1}{6} . \\
\text { For } k=2, Y(3)= \\
\frac{-1}{12}\left[\delta(2-1)+Y(1)+\frac{1}{2 !} \sum_{k_{1}=0}^{1} Y\left(k_{1}\right) Y\left(k-1-k_{1}\right)\right. \\
\left.+\frac{1}{3 !} \sum_{k_{2}=0}^{1} \sum_{k_{1}=0}^{k_{2}} Y\left(k_{1}\right) Y\left(k_{2}-k_{1}\right) Y\left(k-1-k_{2}\right)+\cdots\right]=0
\end{gathered}
$$

and then, $Y(3)=0$. For $k=3$ we have:

$$
Y(4)=\frac{-1}{5 \times 4}\left[\delta(3-1)+Y(2)+\frac{1}{2 !} S_{1}+\frac{1}{3 !} S_{2}+\frac{1}{4 !} S_{3}+\cdots\right]
$$

Now, as $Y(2)=\frac{-1}{6}$ and $S_{1}=S_{2}=S_{3}=\cdots=0$, then $Y(4)=\frac{1}{4 \times 5 \times 6}=\frac{1}{120}$

For $k=4$ we have:

$$
Y(5)=\frac{-1}{6 \times 5}\left[\delta(4-1)+Y(3)+\frac{1}{2 !} S_{1}+\frac{1}{3 !} S_{2}+\frac{1}{4 !} S_{3}+\cdots\right]
$$

In this case as $Y(3)=0$ and $S_{1}=S_{2}=S_{3}=\cdots=0$, then $Y(5)=0$.

The lector can see that

$$
S_{1}=\sum_{k_{1}=0}^{3} Y\left(k_{1}\right) Y\left(k-1-k_{1}\right)=Y(0) Y(3)+Y(1) Y(2)+Y(2) Y(1)+Y(3) Y(0)=0
$$

For $k=5$ we have:

$$
\begin{aligned}
Y(6) & =\frac{-1}{7 \times 6}\left[\delta(5-1)+Y(4)+\frac{1}{2 !} S_{1}+\frac{1}{3 !} S_{2}+\frac{1}{4 !} S_{3}+\cdots\right] \\
& =-\frac{1}{7 \times 6}\left[\frac{1}{120}+\frac{1}{2 !} S_{1}+\frac{1}{3 !} S_{2}+\frac{1}{4 !} S_{3}+\cdots\right]
\end{aligned}
$$

Now, we can see:

$$
\begin{aligned}
S_{1} & =\sum_{k_{1}=0}^{4} Y\left(k_{1}\right) Y\left(k-1-k_{1}\right)=Y(0) Y(4)+Y(1) Y(3)+Y(2) Y(2)+Y(3) Y(1)+Y(4) Y(0) \\
& =\frac{-1}{6} \times-\frac{1}{6}=\frac{1}{36}
\end{aligned}
$$

$S_{2}=S_{3}=\cdots=0$ and then

$$
Y(6)=\frac{-1}{7 \times 6}\left[\frac{1}{120}+\frac{1}{2 !} \times \frac{1}{36}\right]=\frac{-8}{21 \times 6 !}
$$

For $k=6$ we have:

$$
\begin{aligned}
Y(7) & =\frac{-1}{8 \times 7}\left[\delta(6-1)+Y(5)+\frac{1}{2 !} S_{1}+\frac{1}{3 !} S_{2}+\frac{1}{4 !} S_{3}+\cdots\right] \\
& =-\frac{1}{8 \times 7}\left[\frac{1}{2 !} S_{1}+\frac{1}{3 !} S_{2}+\frac{1}{4 !} S_{3}+\cdots\right]
\end{aligned}
$$

Here,

$$
\begin{aligned}
S_{1}=\sum_{k_{1}=0}^{5} Y\left(k_{1}\right) Y\left(k-1-k_{1}\right)= & Y(0) Y(5)+Y(1) Y(4)+ \\
& Y(2) Y(3)+Y(3) Y(2)+Y(4) Y(1)+Y(5) Y(0)=0
\end{aligned}
$$


and

$$
S_{2}=\sum_{k_{2}=0}^{5} \sum_{k_{1}=0}^{k_{2}} Y\left(k_{1}\right) Y\left(k_{2}-k_{1}\right) Y\left(k-1-k_{2}\right)=0
$$

and so, $S_{2}=S_{3}=\cdots=0$. Consequently, $Y(7)=0$.

For $k=7$ we have:

$$
\begin{aligned}
Y(8)= & \frac{-1}{9 \times 8}\left[\delta(7-1)+Y(6)+\frac{1}{2 !} S_{1}+\frac{1}{3 !} S_{2}+\frac{1}{4 !} S_{3}+\cdots\right] \\
& -\frac{1}{9 \times 8}\left[\frac{-8}{21 \times 6 !}+\frac{1}{2 !} S_{1}+\frac{1}{3 !} S_{2}+\frac{1}{4 !} S_{3}+\cdots\right]
\end{aligned}
$$

Here

$$
\begin{aligned}
S_{1}= & \sum_{k_{1}=0}^{6} Y\left(k_{1}\right) Y\left(k-1-k_{1}\right)=Y(0) Y(6)+Y(1) Y(5)+Y(2) Y(4) \\
& +Y(3) Y(3)+Y(4) Y(2)+Y(5) Y(1)+Y(6) Y(0)=\frac{-1}{6} \times \frac{1}{120}+\frac{1}{120} \times-\frac{1}{6}=\frac{-1}{360}
\end{aligned}
$$

and

$$
S_{2}=\sum_{k_{2}=0}^{6} \sum_{k_{1}=0}^{k_{2}} Y\left(k_{1}\right) Y\left(k_{2}-k_{1}\right) Y\left(k-1-k_{2}\right)=\frac{-1}{216}
$$

Consequently, $S_{3}=S_{4}=\cdots=0$. Finally,

$$
Y(8)=\frac{-1}{9 \times 8}\left[\frac{-8}{21 \times 6 !}+\frac{1}{2} \times\left(-\frac{1}{360}\right)+\frac{1}{6} \times\left(-\frac{1}{216}\right)\right]=\frac{61}{1632960}
$$

Therefore using (15), the closed form of the solution can be easily written as:

$$
\begin{aligned}
y(x) & =\sum_{k=0}^{n} Y(k) x^{k}=Y(0) x^{0}+Y(1) x^{1}+Y(2) x^{2}+Y(3) x^{3}+Y(4) x^{4}+\cdots \\
& =-\frac{1}{6} x^{2}+\frac{1}{120} x^{4}-\frac{1}{21 \times 6 !} x^{6}+\frac{61}{1632960} x^{8}-\cdots
\end{aligned}
$$

A series solution obtained by Wazwaz [5] and series expansion respectively is

$$
y(x) \cong-\frac{1}{6} x^{2}+\frac{1}{5 \times 4 !} x^{4}-\frac{1}{21 \times 6 !} x^{6}+\frac{122}{81 \times 8 !} x^{8}-\frac{61 \times 67}{495 \times 10 !} x^{10}+\cdots
$$

Table 1 shows the comparison of $y(x)$ obtained by the DTM (method proposed in this work) and those obtained by Wazwaz. The resulting graph of the isothermal gas spheres equation in comparison to the present method and those obtained by Wazwaz is shown in Figure 2.

Example 2 Consider the following problem $y^{\prime \prime}+\frac{2}{x} y^{\prime}+\mathrm{e}^{-y}=0$ subject to $y(0)=y^{\prime}(0)=0$. Multiplying both sides by $x$

$$
x y^{\prime \prime}+2 y^{\prime}+x \mathrm{e}^{-y}=0
$$

As before, using theorems 1-6 we obtain

$$
Y(k+1)=\frac{-1}{(k+2)(k+1)}\left[\delta(k-1)-Y(k-1)+\frac{1}{2 !} S_{1}-\frac{1}{3 !} S_{2}+\frac{1}{4 !} S_{3}-\cdots\right]
$$

where $S_{1}, S_{2}$ and $S_{3}$ are as (18), (19) and (20) respectively for all $k \geq 1$. Now, from the initial conditions $y(0)=y^{\prime}(0)=0$ we have

$$
Y(0)=0 \text { and } Y(1)=0
$$




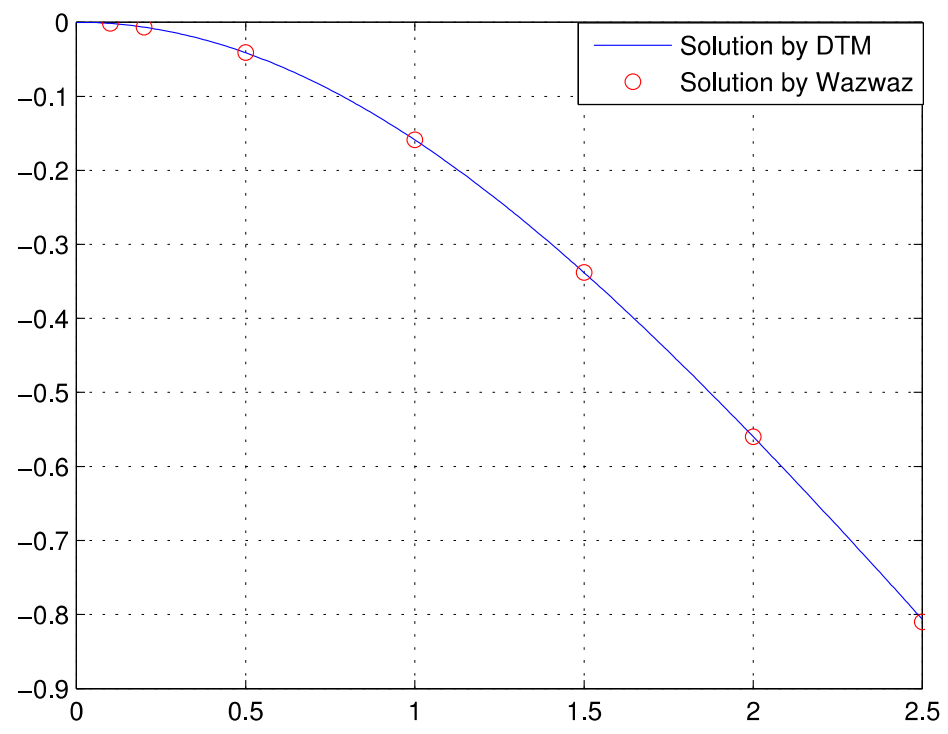

Figure 2. Comparison between DTM and Wazwaz's algorithm.

Table 1. Comparison between DTM and Wazwaz’s algorithm.

\begin{tabular}{cccc}
\hline $\boldsymbol{x}$ & DTM & Wazwaz & Error \\
\hline 0.0 & 0.0000000000 & 0.0000000000 & 0.0000000000 \\
0.1 & -0.0016658338 & -0.0016658338 & 0.0000000000 \\
0.2 & -0.0066533671 & -0.0066533671 & 0.0000000000 \\
0.5 & -0.0411539573 & -0.0411539568 & 0.0000000005 \\
1.0 & -0.1588276775 & -0.1588273536 & 0.0000003239 \\
1.5 & -0.3380194248 & -0.3380131102 & 0.0000063146 \\
2.0 & -0.5598230174 & -0.5599626601 & 0.0001396427 \\
2.5 & -0.8063552923 & -0.8100196713 & 0.0036643790 \\
\hline
\end{tabular}

Substituting Equation (25) into Equation (24) and by recursive method, the results are listed as follows. For $k=1,2,3,4,5$, we have respectively

$$
Y(2)=-\frac{1}{6}, Y(3)=0, Y(4)=-\frac{1}{120}, Y(5)=0, Y(6)=-\frac{1}{1890}
$$

So on, we can use (15) and the closed form of the solution can be easily written as

$$
\begin{aligned}
y(x) & =\sum_{k=0}^{n} Y(k) x^{k}=Y(0) x^{0}+Y(1) x^{1}+Y(2) x^{2}+Y(3) x^{3}+Y(4) x^{4}+\cdots \\
& =-\frac{1}{6} x^{2}-\frac{1}{120} x^{4}-\frac{1}{1890} x^{6}-\cdots
\end{aligned}
$$

A solution obtained by Yahya [12] by using the power series method is

$$
y(x) \cong-\frac{1}{6} x^{2}-\frac{1}{120} x^{4}-\frac{8}{21 \times 6 !} x^{6}-\frac{122}{81 \times 8 !} x^{8}-\cdots
$$

We can see Figure 3 and compare with [13], the results are very good.

As final example and to illustrate the ability of DTM for white-dwarf equation, the next problem is provided for $c=0.2$. 


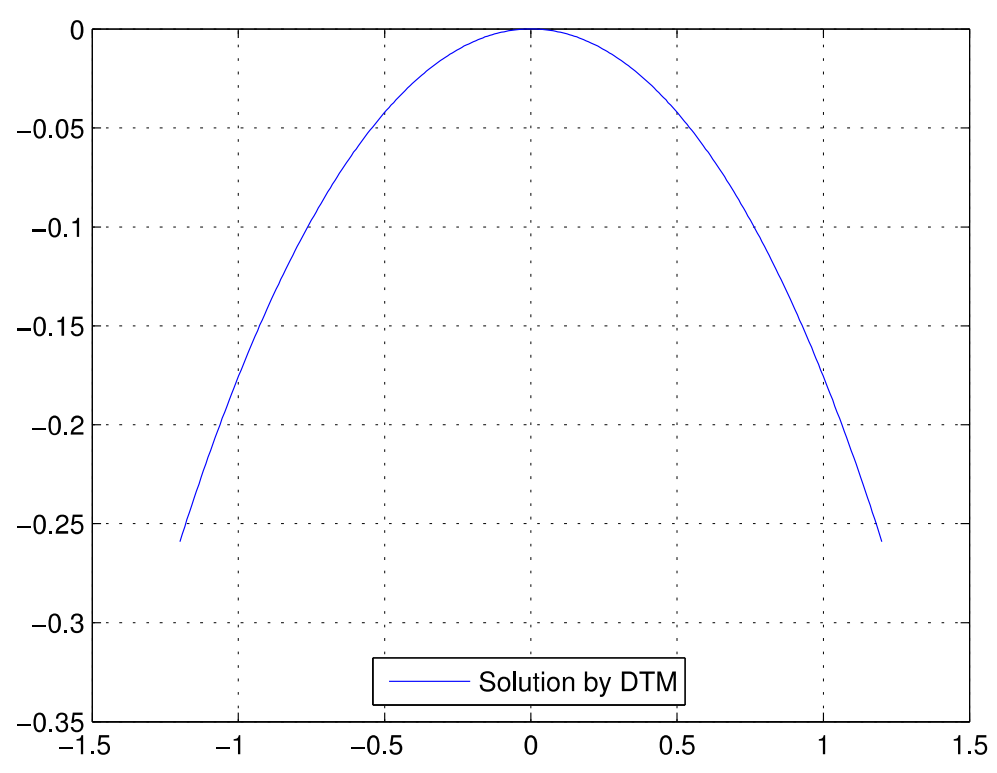

Figure 3. Numerical results by using DTM.

Example 3 Consider the problem $y^{\prime \prime}+\frac{2}{x} y^{\prime}+\left(y^{2}-c\right)^{3 / 2}=0$ subject to $y(0)=1$ and $y^{\prime}(0)=0$. Multiplying both sides by $x$ we obtain

$$
x y^{\prime \prime}+2 y^{\prime}+x\left(y^{2}-c\right)^{3 / 2}=0
$$

Here, is easy to verify that the function $g(y)=\left(y^{2}-c\right)^{3 / 2}$ has a series expansion

$$
g(y) \approx q^{3}+3 q(y-1)+\frac{3 q^{2}+3}{2 q}(y-1)^{2}+\frac{9 q^{2}-3}{3 ! q^{3}}(y-1)^{3}+\cdots
$$

where $q^{2}=1-c$. Therefore, Equation (27) takes the form

$$
x y^{\prime \prime}+2 y^{\prime}+x\left[q^{3}+3 q(y-1)+\frac{3 q^{2}+3}{2 q}(y-1)^{2}+\frac{9 q^{2}-3}{3 ! q^{3}}(y-1)^{3}+\cdots\right]
$$

Using in (29) the above theorems we have the following

$$
(k+1) k Y(k+1)+2(k+1) Y(k+1)+\alpha_{1} \delta(k-1)+\alpha_{2} Y(k-1)+\frac{\alpha_{3}}{2 !} S_{1}+\frac{\alpha_{4}}{3 !} S_{2} \cdots
$$

or

$$
Y(k+1)=\frac{1}{(k+2)(k+1)}\left[-\alpha_{1} \delta(k-1)-\alpha_{2} Y(k-1)-\frac{\alpha_{3}}{2 !} S_{1}-\frac{\alpha_{4}}{3 !} S_{2} \cdots\right]
$$

where

$$
\begin{aligned}
& \alpha_{1}=q^{3}-3 q+\frac{3 q^{2}+3}{2 q}-\frac{9 q^{2}-3}{3 ! q^{3}}+\cdots \\
& \alpha_{2}=3 q-2 \frac{3 q^{2}+3}{2 q}+3 \frac{9 q^{2}-3}{3 ! q^{3}}+\cdots \\
& \alpha_{3}=\frac{3 q^{2}+3}{2 q}-3 \frac{9 q^{2}-3}{3 ! q^{3}}+\cdots
\end{aligned}
$$


and successively. Also,

$$
\begin{gathered}
S_{1}=\sum_{k_{1}=0}^{k-1} Y\left(k_{1}\right) Y\left(k-1-k_{1}\right) \\
S_{2}=\sum_{k_{2}=0}^{k-1} \sum_{k_{1}=0}^{k_{2}} Y\left(k_{1}\right) Y\left(k_{2}-k_{1}\right) Y\left(k-1-k_{2}\right) \\
S_{3}=\sum_{k_{3}=0}^{k-1} \sum_{k_{2}=0}^{k_{3}} \sum_{k_{1}=0}^{k_{2}} Y\left(k_{1}\right) Y\left(k_{2}-k_{1}\right) Y\left(k_{3}-k_{2}\right) Y\left(k-1-k_{3}\right)
\end{gathered}
$$

for all $k \geq 1$. Now, from the initial conditions we have

$$
Y(0)=1 \text { and } Y(1)=0
$$

Substituting (35) into Equation (31) and by recursive method, the results are listed as follows.

For $k=1, Y(2)=\frac{1}{6}\left[-\alpha_{1}-\alpha_{2}-\frac{\alpha_{3}}{2 !}\right]=-\frac{1}{6} q^{3} . \quad$ or $\quad k=2, Y(3)=\frac{1}{12}\left[-\alpha_{1} \delta(2-1)-\alpha_{2} Y(1)-\frac{\alpha_{3}}{2 !} S_{1}-\cdots\right]=0$ and then $Y(3)=0$. for $k=3, Y(4)=\frac{1}{20}\left[-\alpha_{1} \delta(3-1)-\alpha_{2} Y(2)-\frac{\alpha_{3}}{2 !} S_{1}-\cdots\right]$ and then $Y(4)=\frac{1}{40} q^{4}$. For $k=4, Y(5)=\frac{1}{30}\left[-\alpha_{1} \delta(4-1)-\alpha_{2} Y(3)-\frac{\alpha_{3}}{2 !} S_{1}-\cdots\right]$ and so $Y(5)=0$. For $k=5, Y(6)=\frac{1}{42}\left[-\alpha_{1} \delta(5-1)-\alpha_{2} Y(4)-\frac{\alpha_{3}}{2 !} S_{1}-\cdots\right]$ therefore $Y(6)=-\frac{5}{5040} q^{7}-\frac{14}{5040} q^{5}$. Using (15), the closed form of the soluyion can be easily written as

$$
\begin{aligned}
y(x) & =\sum_{k=0}^{n} Y(k) x^{k}=Y(0) x^{0}+Y(1) x+Y(2) x^{2}+Y(3) x^{3}+\cdots \\
& =1-\frac{1}{6}(1-c)^{3 / 2} x^{2}+\frac{1}{40}(1-c)^{4} x^{4}-\left(\frac{5}{5040}(1-c)^{7 / 2}+\frac{14}{5040}(1-c)^{5 / 2}\right) x^{6}+\cdots
\end{aligned}
$$

A series solution obtained by Chandrasekhar [2] using series expansion was

$$
y(x) \cong 1-\frac{q^{3}}{6} x^{2}+\frac{q^{4}}{40} x^{4}-\frac{q^{5}}{7 !}\left(5 q^{2}+14\right) x^{6}+\frac{q^{6}}{3 \times 9 !}\left(339 q^{2}+280\right) x^{8}+\cdots
$$

Table 2 shows the comparison of $y(x)$ obtained by the DTM and those obtained by Parand [14]. The resulting graph of the white-dwarfs equation in comparison to the present method and the obtained by [14] is show in Figure 4.

\section{On Convergence of DTM}

We can write the DTM as

Table 2. Comparison between DTM and Legendre-Spectral method.

\begin{tabular}{cccc}
\hline$x$ & DTM & L-S method & Error \\
\hline 0.0 & 0.0000000000 & 0.0000000000 & 0.0000000000 \\
0.5 & 0.9812035800 & 0.912034800 & 0.0000001000 \\
1.0 & 0.9270041568 & 0.9270031568 & 0.0000010000 \\
1.5 & 0.8439248841 & 0.8439247581 & 0.0000001260 \\
2.0 & 0.7425430743 & 0.7425430235 & 0.0000000508 \\
2.5 & 0.6365969111 & 0.6365953025 & 0.0000016086 \\
3.0 & 0.5410635754 & 0.5410633690 & 0.0000002064 \\
3.5 & 0.4691977489 & 0.4691238259 & 0.0000739230 \\
\hline
\end{tabular}




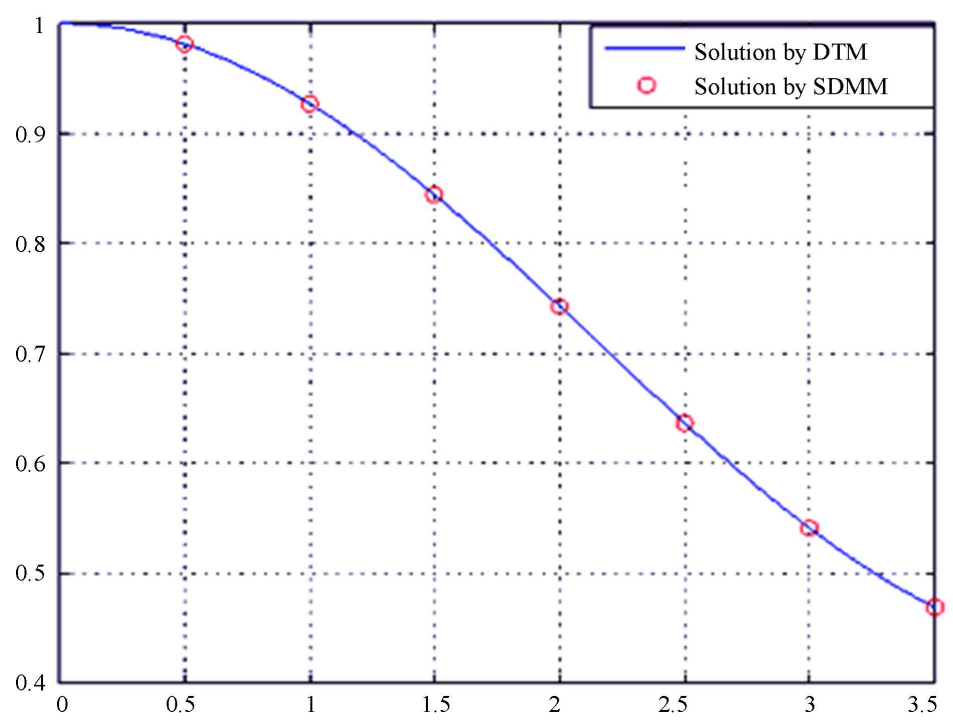

Figure 4. Comparison between DTM and a Legendre-Spectral Method.

$$
\sum_{j=0}^{k} \alpha_{j} y_{n+j}=h \phi_{f}\left(x_{n}, y_{n}, \cdots, y_{n+k-1} ; h\right), n=0, \cdots, N-k
$$

where $\phi_{f}$ increase function depends on its arguments through the function $f$. The method (37) means $k$ steps, needed for early values $k: y_{n}, \cdots, y_{n+k-1}$ to calculate $y_{n+k}$. It is therefore necessary to have bootstrap values $y_{0}, \cdots, y_{k-1}$.

The method (37) is said to be convergent if for all IVP has to

$$
\lim _{N \rightarrow \infty} \max _{k \leq n \leq N}\left\|y\left(x_{n}-y_{n}\right)\right\|=0 \quad \text { if } \lim _{N \rightarrow \infty} \max _{0 \leq n \leq k-1}\left\|y\left(x_{n}-y_{n}\right)\right\|=0
$$

Remark. The condition $\lim _{N \rightarrow \infty} \max _{0 \leq n \leq k-1}\left\|y\left(x_{n}-y_{n}\right)\right\|=0$ on the bootstrap values is equivalent to asking that $\lim _{h \rightarrow 0^{+}} y_{n}=y\left(x_{0}\right)$ for $n=0, \cdots, k-1$. Here, we are asking that bootstrap values $\left\{y_{n}\right\}_{n=0}^{k-1}$ approximate well and the initial data $y\left(x_{0}\right)$; if this is not, then no reason to expect that numerical solution closely matches the theoretical.

Now let us consider the following form of the Equation (1)

$$
\mathcal{G}(y(x))=0
$$

Here $\mathcal{G}$ is a nonlinear differential operator, which encloses the linear and nonlinear term of the Lane-Emden type equation. Now, the linear term $\mathcal{H}=y^{(n)}$ is always invertible and the nonlinear term is $\mathcal{I}$ Therefore (38) may be written as

$$
\mathcal{H}(y(x))+\mathcal{J}(y(x))=0
$$

or

$$
\mathcal{H}(y(x))=-\mathcal{J}(y(x))=0
$$

Applying DTM in (40) we can obtain

$$
(k+1)(k+2) \cdots(k+n) Y(k+n)=-\mathfrak{J}(y(x))
$$

Remember that differential transformation of $\mathcal{G}$ and $\mathcal{I}$ are computed by using theorems 1 - 6 .

Let us consider the Equation (38) in the following form

$$
y(x)=\mathcal{Y}(y(x))
$$


Here, $\mathcal{Y}$ is a nonlinear operator. It is noted that Equation (15) is equivalent to the sequence

$$
\sum_{k=0}^{n} Y(k) x^{k}=y_{0}+y_{1}+y_{2}+\cdots+y_{n}=T_{n}
$$

This sequence is determined using the iterative scheme

$$
T_{n+1}=\mathcal{Y}\left(T_{n}\right)
$$

and associated with $T=\mathcal{Y}(T)$.

The following theorem guarantees that the scheme of DTM converges to the solution $y(x)$ of Lane-Emden Equation (1).

Theorem 7 Let $\mathcal{Y}$ be a nonlinear operator from a Banach space $x$ and $y(x)$ be the solution (exact) of Equation (42). The series solution (14) converges to $y(x)$, if there exists a constant $0 \leq c<1$ such that $\left\|y_{k+1}\right\| \leq c\left\|y_{k}\right\|$ for $k \in \mathbb{Z}^{+} \bigcup\{0\}$.

Proof. We prove that the sequence $\left\{T_{n}\right\}_{n=0}^{\infty}$ is a Cauchy sequence in $X$. Therefore,

$$
\left\|T_{n}-T_{n+1}\right\|=\left\|y_{n+1}\right\| \leq c\left\|y_{n}\right\| \leq c^{2}\left\|y_{n-1}\right\| \leq \cdots \leq c^{n+1}\left\|y_{0}\right\|
$$

Thus, for any $m, n \in \mathbb{Z}^{+}, m \leq n$,

$$
\begin{aligned}
\left\|T_{n}-T_{m}\right\| & \leq\left\|T_{n}-T_{n-1}\right\|+\left\|T_{n-1}-T_{n-2}\right\|+\cdots+\left\|T_{m+1}-T_{n}\right\| \\
& \leq c^{n}\left\|y_{0}\right\|+c^{n-1}\left\|y_{0}\right\|+\cdots+c^{m+1}\left\|y_{0}\right\| \\
& \leq\left(c^{m+1}+c^{m+2}+\cdots+c^{n}\right)\left\|y_{0}\right\| \\
& =c^{m+1} \frac{1-c^{n-m}}{1-c}\left\|y_{0}\right\|
\end{aligned}
$$

so $\left\|T_{n}-T_{m}\right\|<\frac{c^{m+1}}{1-c}\left\|y_{0}\right\|$ implying that he sequence $\left\{T_{n}\right\}_{n=0}^{\infty}$ is Cauchy, i.e. since $0 \leq c<1$ then $\lim _{n, m \rightarrow \infty}\left\|T_{n}-T_{m}\right\|=0$, therefore there exists $T \in \mathcal{X}$ such that $\lim _{n \rightarrow \infty} T_{n}=T$, i.e. $T=\sum_{n=0}^{\infty} y_{n}$ converges.

Now, we can say too that Equation (42) is similar to solve $T=\mathcal{Y}(T)$, therefore this implies that if $\mathcal{Y}$ is continuous then

$$
\mathcal{Y}(T)=\mathcal{Y}\left(\lim _{n \rightarrow \infty} T_{n}\right)=\lim _{n \rightarrow \infty} \mathcal{Y}\left(T_{n}\right)=\lim _{n \rightarrow \infty}\left(T_{n+1}\right)=T
$$

i.e. $T$ is a solution of $y(x)=\mathcal{Y}(y(x))$ and this completes the proof.

Figure 5 shows the maximum point-wise error between the numerical solution obtained by using DTM and the Chandrasekhar solution. It is observed that both schemes are almost the same accuracy.

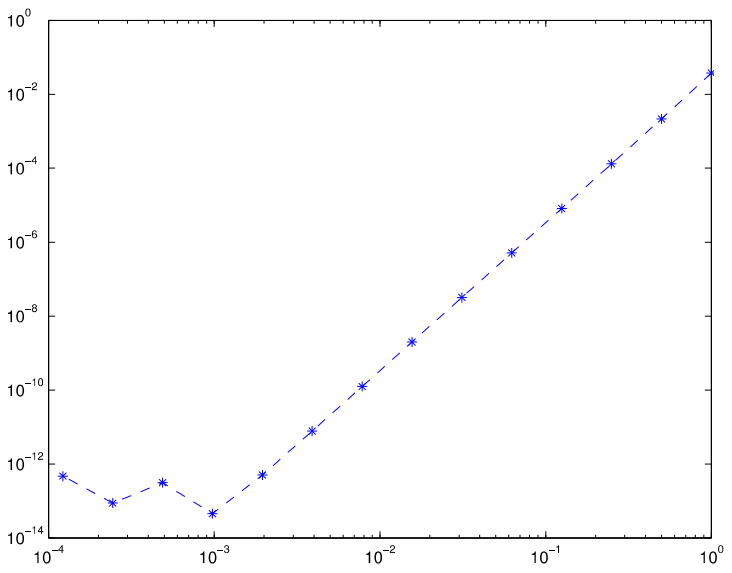

Figure 5. Example 1. $\left\|e_{\infty}\right\|$ error obtained by using DTM. 


\section{Conclusion}

In this work, we presented the definition and handling of one-dimensional differential transformation method. Using DTM, the Lane-Emden equations were transformed into algebraic equations (iterative equations). The new scheme obtained by using DTM yields an analytical solution in the form of a rapidly convergent series. This method makes the solution procedure much more attractive. The figures and tables clearly show the high efficiency of DTM and the convergence of the method for three examples in investigated.

\section{Acknowledgements}

Foremost, I would like to express my sincere gratitude to Jean-Christophe Nave (Department of Mathematics and Statics McGill University) for the support of my research and the support of the Department of Mathematics of the Universidad Tecnológica de Pereira (Colombia) and the group GEDNOL.

\section{References}

[1] Davis, H. (1962) Introduction to Nonlinear Differential and Integral Equations. Dover, New York.

[2] Chandrasekhar, S. (1967) Introduction to Study of Stellar Structure. Dover, New York.

[3] Liao, S.J. (2003) A New Analytic Algorithm of Lane-Emden Type Equations. Advances in Applied Mathematics, 142, 1-16. http://dx.doi.org/10.1016/S0096-3003(02)00943-8

[4] Zhou, J.K. (1986) Differential Transformation and Its Applications for Electrical Circuits. Huazhong University Press, Wuhan.

[5] Wazwaz, A.M. (2001) A New Algorithm for Solving Differential Equations of Lane-Emden Type. Applied Mathematics and Computation, 118, 287-310. http://dx.doi.org/10.1016/S0096-3003(99)00223-4

[6] Gorder, R.A. (2011) An Elegant Perturbation Solution for the Lane-Emden Equation of the Second Kind. New Astronomy, 16, 65-67. http://dx.doi.org/10.1016/j.newast.2010.08.005

[7] Ramos, J.I. (2008) Series Approach to the Lane-Emden Equation and Comparison with the Homotopy Perturbation Method. Chaos Solitons Fractals, 38, 400-408. http://dx.doi.org/10.1016/j.chaos.2006.11.018

[8] Iqbal, S. and Javed, A. (2005) Application of Optimal. Advances in Applied Mathematics, 42, 29-48.

[9] Kumar, N. and Pandey, R. (2011) Solution of the Lane-Emden Equation Using the Bernstein Operational Matrix of Integration. ISRN Astronomy and Astrophysics, 2011, 1-7. http://dx.doi.org/10.5402/2011/351747

[10] Rismani, A.M. and Monfared, H. (2102) Numerical Solution of Singular IVPs of Lane-Emden Type Using a Modified Legendre-Spectral method. Applied Mathematical Modelling, 36, 4830-4836. http://dx.doi.org/10.1016/j.apm.2011.12.018

[11] Cárdenas, P. and Arboleda, A. (2012) Resolución de ecuaciones diferenciales no lineales por el método de transfor-mación diferencial, tesis de maestría en matemáicas. Universidad Tecnológica de Pereira, Colombia.

[12] Yahya, H. (2012) On the Numerical Solution of Lane-Emden Type Equations. Advances in Computational Mathematics and Its Applications (ACMA), 4, 191-199.

[13] Batiha, B. (2009) Numerical Solution of a Class of Singular Second-Order IVPs by Variational Iteration Method. International Journal of Mathematical Analysis, 3, 1953-1968. http://www.m-hikari.com/ijma/ijma-password-2009/ijma-password37-40-2009/batihaIJMA37-40-2009.pdf

[14] Parand, K. and Hojjati, G. (2011) An Efficient Computational Algorithm for Solving the Nonlinear Lane-Emden Type Equations. World Academy of Science, Engineering and Technology, 56, 889-894.

http://www.waset.org/publications/11056 